\title{
DESPERATELY SEEKING THE STANDARD MODEL
}

\author{
CARLOS MUÑOZ \\ Departamento de Fúsica Teórica $C$-XI and Instituto de Fúsica Teórica C-XVI, \\ Universidad Autónoma de Madrid, Cantoblanco, 28049 Madrid, Spain. \\ E-mail: carlos.munnoz@uam.es
}

In the mid eighties string phenomenology started. Since then, its main objective, the search of the standard model, has not been accomplished yet. In this talk, on the ocassion of the 2nd International Conference on String Phenomenology in 2003, I will review this crucial issue.

\section{Introduction}

Since this is the last talk of this meeting, and everybody is already exhausted, following the suggestion of the organizers I will try to give an 'entertaining' talk about string phenomenology. To tell you the truth, I do not know whether something concerning string phenomenology can be entertaining for an audience. In any case, please do not take too seriously all things that I am going to say. Some of them are jokes!, or perhaps exaggerations.

The outline of the talk is very simple. Basically, it is divided in two parts. The first one is very brief and I will give an optimistic view about string theory and phenomenology. Following Dyson's analogy between the quantum field theory and the 19th-century chemistry-both explain how but not why-one could also establish an analogy between atomic physics and string theory. Atomic physics was needed to answer the question why in chemistry, string theory is supposed to answer the question why in the standard model of particle physics. I will review this attempt in the second part of this talk. In this sense, in that part I will give a more realistic view of string phenomenology. Perhaps, some of you in the audience will consider this view slightly pessimistic. Let us see!

\section{Optimistic View}

Dyson, in an article written in $1953^{1}$, drew the following analogy between the quantum field theory and the 19th-century chemistry: 'The latter described the properties of the chemical elements and their interactions. How the elements behave; it did not try to explain why a particular set of elements, each with its particular properties, exists. To answer the question why, completely new sciences were needed: atomic and nuclear physics. (...) The quantum 
Table 1. Chart of the fundamental particles (1953) listed in the order of their mass.

\begin{tabular}{|c|}
\hline photon \\
\hline graviton \\
\hline neutrino \\
\hline electron \\
\hline positron \\
\hline positive mu meson \\
\hline negative mu meson \\
\hline neutral pi meson \\
\hline positive pi meson \\
\hline negative pi meson \\
\hline zeta meson ? \\
\hline neutral V-particle \\
\hline tau meson \\
\hline kappa meson \\
\hline positive chi meson \\
\hline negative chi meson \\
\hline proton \\
\hline neutron \\
\hline neutral V-particle \\
\hline positive V-particle ? \\
\hline
\end{tabular}

field theory treats elementary particles just as 19th-century chemists treated the elements. The theory is in its nature descriptive and not explanatory. It starts from the existence of a specified list of elementary particles, with specified masses, spins, charges and specified interactions with one another. All these data are put into the theory at the beginning. The purpose of the theory is simply to deduce from this information what will happen if particle $A$ is fired at particle $B$ with a given velocity. We are not yet sure whether the theory will be able to fulfill even this modest purpose completely. Many technical difficulties have still to be overcome. One of the difficulties is that we do not yet have the complete list of elementary particles (see Table 11). Nevertheless the successes of the theory in describing experimental results have been striking. It seems likely that the theory in something like its present form will describe accurately a very wide range of possible experiments. This is the most that we would wish to claim for it'.

Now, in 2003, as shown in Table 2 we do have the complete list of elementary particles (at least at energies below the electroweak scale, and given some uncertainties related to the Higgs sector)-the proton, pi meson, etc. which appeared in the chart of fundamental particles in Dyson's presentation should be regarded as an amusing historical anecdote-and we do know that 
Table 2. Chart of the fundamental particles in 2003 (modification of Table 1 taking into account the new experimental results since 1953).

\begin{tabular}{|c|}
\hline photon \\
\hline graviton \\
\hline neutrino $(\mathbf{s})$ \\
\hline electron \\
\hline positron \\
\hline positive mu meson \\
\hline negative mu meson \\
\hline neutral pi meson (non elementary) \\
\hline positive pi meson ( non elementary) \\
\hline negative pi meson (non elementary) \\
\hline zeta meson ? (non elementary) \\
\hline neutral V-particle ( non elementary) \\
\hline (positive and negative) tau meson \\
\hline kappa meson (non elementary) \\
\hline positive chi meson (non elementary) \\
\hline negative chi meson ( non elementary) \\
\hline proton (non elementary) \\
\hline neutron ( non elementary) \\
\hline neutral V-particle (non elementary) \\
\hline positive V-particle? (non elementary) \\
\hline gluons \\
\hline quarks + antiquarks \\
\hline $\mathbf{W}^{ \pm}, \mathbf{Z}^{0}$ \\
\hline Higgs ? \\
\hline
\end{tabular}

the theory fulfilling the modest purpose mentioned by Dyson is the standard model 2 .

What the 19th-century chemistry did with the chemical elements, the 20th-century standard model does with the elementary particles. It describes how the elementary particles behave but does not try to explain why a particular set of elementary particles, each with its particular properties, exists. To answer the question why it seems that new sciences, as atomic and nuclear physics in the case of chemistry, are not needed, but just new theories. This is precisely one of the purposes of string theory (as a matter of fact, originally, the main purpouse of the modern string theory was "simply" to unify all gauge interactions with gravity ${ }^{3}$ in a consistent way 4 ).

As is well known, in string theory the elementary particles are not pointlike objects but extended, string-like objects. It is still surprising that this apparently small change allows us to answer fundamental questions that in the context of the quantum field theory of point-like particles cannot even 
Figure 1. The three great leaders trying to convince us that the weapons of mass destruction exist.

be posed. For example: Why is the standard model gauge group $S U(3) \times$ $S U(2)_{L} \times U(1)_{Y}$ ? Why are there three families of particles? Why is the mass of the electron $m_{e}=0.5 \mathrm{MeV}$ ? Why is the fine structure constant $\alpha=1 / 137$ ?

In this sense one can have the temptation of thinking that the comment about chemists that Dyson wrote in his article - Looking backward, it is now clear that 19th-century chemists were right to concentrate on the how and to ignore the why. They did not have the tools to begin to discuss intelligently the reasons for the individualities of the elements. They had to spend a hundred years building up a good quantitative descriptive theory before they could go further. And the result of their labors, the classical science of chemistry, was not destroyed or superseded by the later insight that atomic physics gave.'will be written similarly by somebody in the future about $20 / 21$ th-century physicists, substituting elements by elementary particles, science of chemistry by standard model and atomic physics by string theory.

Of course, let us hope that in this case the task will be accomplished before a hundred years since the standard model started to be built. Otherwise, many of the people following this talk (including the speaker) will be probably dead and buried!

\section{Realistic (Pessimistic?) View}

What is string phenomenology? A possible answer to this question is to say that string phenomenology is the search of the standard model in string theory. Of course, this is not the only task, but clearly to found the standard model is a necessary condition in string phenomenology. It would be a little bit annoying to explain the big bang singularity using strings but not to be able to obtain the standard model! In this sense, is is fair to say that almost 20 years have gone by since string phenomenology started, and the standard model has not been found yet.

Now, is this really a big problem? Perhaps, in order to answer this question, we should get some inspiration from the three great leaders shown in Fig. 1 They have not found the weapons of mass destruction yet, but they want us to believe that they will found them in a few months. In the same way, we have not found the standard model yet, but we want the people to believe that we will find it in a few years.

paper: submitted to World Scientific on January 3, 2019 
More seriously, let us briefly review the history of string phenomenology concerning the search of the standard model. May be, in this way, we will be able to have a more clear opinion about whether the (string) standard model can be found in the near future. In a sense, the compactification of the tendimensional heterotic string 5 on six-dimensional spaces might be consider as the starting point for this race $\underline{6}$. In particular, Calabi-Yau spaces $\underline{6}$, orbifolds 7 and fermionic constructions $\frac{8}{8}$ proved to be interesting methods to carry out the task. It was shown pretty soon that these compactifications of the $E_{8} \times E_{8}$ heterotic string can give rise to four-dimensional standard-like models as well as GUT-like models 9-11] Clearly, these results were extremely interesting. Since then we know that (at least) something close to the real world can arise from strings.

For the sake of concreteness, let us review the case of orbifolds, without entering into many mathematical details or technicalities. It was first shown that the use of discrete Wilson lines 7112 on the torus defining a symmetric orbifold can give rise to four-dimensional supersymmetric models with gauge group $1314 S U(3) \times S U(2) \times U(1)^{5} \times G_{\text {hidden }}$. In addition, it was also shown that three generations of chiral particles (plus extra particles) appear in a natural way using just two Wilson lines. In fact this result was obtained in the case of the $Z_{3}$ orbifold. The latter is constructed by dividing $R^{6}$ by the $[S U(3)]^{3}$ root lattice modded by the point group $(P)$ with generator $\theta$, where the action of $\theta$ on the lattice basis is $\theta e_{i}=e_{i+1}, \theta e_{i+1}=-\left(e_{i}+e_{i+1}\right)$, with $i=1,3,5$. The two-dimensional sublattices associated to $[S U(3)]^{3}$ are shown in Fig. 2 In orbifold constructions, twisted strings appear attached to fixed points under the point group. In the case of the $Z_{3}$ orbifold there are 27 fixed points under $P$, and therefore there are 27 twisted sectors. We will denote the three fixed points of each two-dimensional sublattice as shown in Fig. 2 Thus the three generations arise because in addition to the overall factor of 3 coming from the right-moving part of the untwisted matter, the twisted matter come in 9 sets with 3 equivalent sectors on each one. Let us suppose that the two Wilson lines correspond to the first and second sublattices The three generations correspond to move the third sublattice component $(\mathrm{x} \cdot \mathrm{o})$ of the fixed point keeping the other two fixed.

The next step was the calculation of the $U(1)$ charges and the study of the mechanism for anomaly cancellation in these models 15 , since an anomalous $U(1)$ is usually present after compactification 16. This allowed the construction of combinations of the non-anomalous $U(1)$ 's giving the physical hypercharge for the particles of the standard model, although it was also found that the hidden sector is, in general, mixed with the observable one through the 


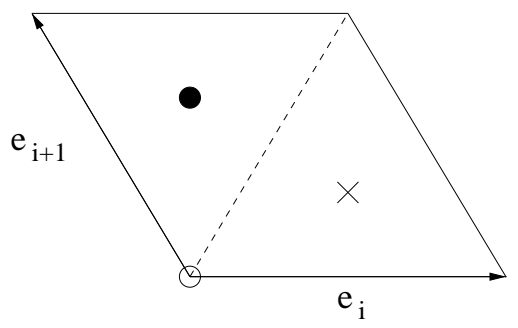

Figure 2. Two dimensional sublattices $(i=1,3,5)$ of the $Z_{3}$ orbifold. The fixed point components are also shown.

extra $U(1)$ charges. Fortunately, it was also noted that the Fayet-Iliopoulos D-term 16, which appears because of the presence of the anomalous $U(1)$, can give rise to the breaking of the extra $U(1)$ 's and, as a consequence, to the hiding of the previously mixed hidden sector 1510. This is because, in order to preserve supersymmetry at high energies, some scalars with $U(1)$ 's quantum numbers acquire large vacuum expectation values (VEVs). In this way it was possible to construct supersymmetric models 10 (or, more precisely, vacuum states) where the original $S U(3) \times S U(2) \times U(1)^{5} \times S O(10) \times U(1)^{3}$ gauge group 13 was broken to $S U(3) \times S U(2) \times U(1)_{Y} \times S O(10)_{\text {hidden }}$. In addition, the extra particles are highly reduced since many of them get a high mass $\left(\approx 10^{16-17} \mathrm{GeV}\right)$ through the Fayet-Iliopoulos mechanism, thus disappearing from the low-energy theory.

But...is a model with the gauge group of the standard model and three families of quark and leptons, the sought-after standard model? By no means! For this the right model must reproduce also the correct mass hierarchy for quarks and leptons. For example, to obtain

$$
\frac{m_{t}}{m_{u}} \sim 10^{5}, \quad \frac{m_{\tau}}{m_{e}} \sim 10^{3},
$$

is not a trivial task, although it is true that one can find interesting results in the literature concerning this point. In particular, orbifold spaces have a beautiful mechanism to generate a mass hierarchy at the renormalizable level. Namely, Yukawa couplings between twisted matter can be explicitly computed and they get suppression factors, which depend on the distance between the fixed points to which the relevant fields are attached $17-20$. The couplings can be schematically written as

$$
\lambda \sim e^{-\sum_{i} c_{\lambda}^{i} T_{i}}, \quad \operatorname{Re} T_{i} \sim R_{i}^{2},
$$


where the $T_{i}$ are the moduli fields associated to the size and shape of the orbifold. The distances can be varied by giving different VEVs to these moduli, implying that one can span in principle five orders of magnitude the Yukawa couplings 1920 .

Unfortunately, this is not the end of the story. As if to obtain this hierarchy were not difficult enough, Nature is even more cruel with string phenomenologists. It tells us that a weak coupling matrix exists 21 with weird magnitudes for the entries 22

$$
V_{C K M}=\left(\begin{array}{cccc}
0.9741 \text { to } 0.9756 & 0.219 \text { to } 0.226 & 0.0025 \text { to } 0.0048 \\
0.219 \text { to } 0.226 & 0.9732 \text { to } 0.9748 & 0.038 \text { to } 0.044 \\
0.004 \text { to } 0.014 & 0.037 \text { to } 0.044 & 0.9990 \text { to } 0.9993
\end{array}\right),
$$

and that therefore we must arrange our up-and down-quark Yukawa couplings in order to have specific off diagonal elements,

$$
H_{2}^{0} \bar{u}_{L \alpha} \lambda_{u}^{\beta \gamma} u_{R \gamma}+H_{1}^{0} \bar{d}_{L \alpha} \lambda_{d}^{\beta \gamma} d_{R \gamma} .
$$

In principle this property arises naturally in orbifolds $18-2023$. For example, in the $Z_{3}$ orbifold with two Wilson lines, if the $\mathrm{SU}(2)$ doublet $H_{2}$ corresponds to (o o o), the three generations of $(3,2)$ quarks to $(\mathrm{o} o(\mathrm{o}, \mathrm{x}, \cdot)$ ) and the three generations of $(\overline{3}, 1)$ up-quarks to $(\mathrm{o} o(\mathrm{o}, \mathrm{x}, \cdot))$, then there are three couplings allowed from the space group selection rule (the components of the three fixed points in each sublattice must be either equal or different): $\lambda_{t t} H_{2}^{0} \bar{t}_{L} t_{R}$ associated to (o o o)(o o o)( о о o) with $\lambda_{t t} \sim 1, \lambda_{c u} H_{2}^{0} \bar{c}_{L} u_{R}$ associated to (o o o)(o o x $)($ o o $\cdot)$ with $\lambda_{c u} \sim e^{-T_{5}}$, and $\lambda_{u c} H_{2}^{0} \bar{u}_{L} c_{R}$ associated to $(\mathrm{o}$ o o $)(\mathrm{o} \circ \cdot)(\mathrm{o} \circ \mathrm{x})$ with $\lambda_{u c} \sim e^{-T_{5}}$. In this simple example one gets one diagonal Yukawa coupling without suppression factor and two off diagonal degenerate ones $\sim e^{-T_{5}}$, but other more realistic examples producing the observed structure of quark and lepton masses and mixing angles can be obtained using three generations of Higgses 20 or three Wilson lines 19.

Unfortunately, although the mechanisms discussed above are attractive, it is extremely difficult to implement them in a particular model. Given a model, everything is essentially fixed, and it is not possible to play around. For example, one can have a model with the coupling for the bottom allowed but not for the top, or with both forbidden, or with no element 13 in the CKM matrix (3), or... The truth is that no model has been found with all the necessary Yukawa couplings. As a matter of fact, not even a model close to obtain them. And this sentence can also be applied to any of the interesting models constructed in more recent years 2425 .

In this sense, in my opinion the main difficulty in string model building resides in how to obtain the weird structure of fermion masses and mixing

paper: submitted to World Scientific on January 3, 2019 
angles. My good friend and old collaborator Alberto Casas always says, 'I wouldn't mind to die once I knew the mechanism generating the CKM matrix'. Thus, please, do not find it too soon, we want him to stay alive, at least for a while.

Needless to say, the recent experimental confirmation of neutrino masses makes this task even more involved. Now, in addition to hierarchies such as those shown in eq. (11), we have to explain others such as

$$
\frac{m_{e}}{m_{\nu}} \gtrsim 10^{6}
$$

and in addition to the matrix (31), we have to explain the weak coupling matrix 26 with the charged leptons 27

$$
V_{M N S}=\left(\begin{array}{cccc}
0.73 \text { to } 0.89 & 0.45 \text { to } 0.66 & <0.24 \\
0.23 \text { to } 0.66 & 0.24 \text { to } 0.75 & 0.52 \text { to } 0.87 \\
0.06 \text { to } 0.57 & 0.40 \text { to } 0.82 & 0.48 \text { to } 0.85
\end{array}\right) .
$$

Again, as usual in string theory, one can find interesting mechanisms to try to explain these experimental results. For example, if the Yukawa coupling for the neutrino is of order $m_{e}$ and the see saw scale is $1 \mathrm{TeV}$, then the expected neutrino mass is

$$
\frac{m_{e}^{2}}{1 T e V}=0.25 \mathrm{eV},
$$

which is within an order of magnitude of the experimental values. This suggests that a natural situation is one in which a see-saw mass of order a few $\mathrm{TeV}$ is generated by the electroweak symmetry breaking. The first guess for the neutrino see-saw superpotential is then 20

$$
W_{\nu} \sim \lambda_{\nu} H_{2}^{0} L_{L} \nu_{R}+\lambda_{N} N \nu_{R} \nu_{R}
$$

where $N$ is the same singlet that dynamically generates the $\mu$ term through the coupling ${ }^{a} N H_{2}^{0} H_{1}^{0}$. Therefore $N$ is expected to get a VEV of order $1 \mathrm{TeV}$, and the coupling $\lambda_{\nu}$ is expected to be sufficiently small as to reproduce the neutrino mass. Since small couplings can be naturally obtained in orbifolds as discussed in eq. (2), this mechanism is in principle interesting. But recall: to implement any mechanism in a particular model is highly non-trivial.

However, since we are optimistic people, we can argue that if the standard model arises from strings (something that we believe firmly!) there must exist one model where the above mechanism can be implemented, producing the

${ }^{a}$ Note in this context that the Giudice-Masiero mechanism to generate a $\mu$ term through the Kähler potential is not available for prime orbifolds such as the $Z_{3}$ orbifold. 
Figure 3. Two transparencies summarizing the situation concerning the search of the standard model in string phenomenology in 1990 (still can be used in 2003!).

correct structure for Yukawas. This means a model with: 1) the necessary Yukawas couplings, $\left.H_{2}^{0} \bar{u}_{L} \lambda_{u u} u_{R}+H_{2}^{0} \bar{u}_{L} \lambda_{u c} c_{R}+H_{1}^{0} \bar{d}_{L} \lambda_{d b} b_{R}+\ldots, 2\right)$ the correct values, i.e. at least one is able to put by hand the values of $T_{i}$ such that $\lambda_{t}\left(T_{i}\right) \sim 1, \lambda_{u}\left(T_{i}\right) \sim 10^{-5}$, etc. If, at the end of the day, such a model exists this would be a great success. But, in order to be sure that this is really the superstring standard model one should be able to compute explicitly the values of the Yukawas, and for this we need to know the VEVs of the $T_{i^{-}}$ moduli. Unfortunately, these are related to the breaking of supersymmetry, and this is one of the biggest problems in string theory ${ }^{b}$. It is true that there are candidates for this task, such as gaugino condensation in a hidden sector with a non-perturbative superpotential $W\left(S, T_{i}\right)$, and that we have hidden gauge groups that could condensate. However, again, implementing this mechanism in a particular model is not easy.

The above discussion could be summarized with the two transparencies shown in Fig. 3 What is annoying for me is that I prepared these two transparencies for a meeting in Trieste 28 in 1990 , and still I can use them in this meeting 13 years later! In a sense the problem of string theory is that it is too ambitious: the correct model must reproduce not only the gauge group and families of the standard model, but also the correct values of the gauge couplings, the correct masses of quark and leptons, a realistic CKM matrix, etc., i.e. the more than 20 parameters fixed by the experiment in the standard model.

In addition, there are thousands of models (vacua) that can be built. Some of them have the gauge group of the standard model or GUT groups, three families of particles, and other interesting properties, but many others have a number of families different from three, no appropriate gauge groups, no appropriate matter, etc. A perfect way of solving this problem would be to use a dynamical mechanism to select the correct model (vacuum). Such a mechanism should be able to determine a point in the parameter space of the heterotic string determining the correct compactification with $S U(3) \times$ $S U(2)_{L} \times U(1)_{Y}$, three families of particles, and such that the mechanism

${ }^{b}$ As a matter of fact we should also be able to compute the values of the gauge couplings $g_{3}, g_{2}, g_{1}$, determined by the VEV of the dilaton field $S$. Let us recall that this field arises from the gravitational sector of the theory, and that in string theory there are no free parameters, all coupling constants are in fact no constants but fields.

paper: submitted to World Scientific on January 3, 2019 
of supersymmetry breaking (whatever it is) produces $\left\langle S, T_{i}\right\rangle$ generating the correct values for

$$
g_{3}, g_{2}, g_{1}, \lambda_{u}, \lambda_{d}, \lambda_{c}, \lambda_{s}, \lambda_{t}, \lambda_{b}, \lambda_{e}, \lambda_{\mu}, \lambda_{\tau}, \lambda_{\nu_{e}}, \lambda_{\nu_{\mu}}, \lambda_{\nu_{\tau}}, \delta, \theta_{c}, \ldots
$$

In a sense, it is hard to believe that there exists a (top-bottom) mechanism with such a precision determining everything. But here we apply again our optimism, arguing that the standard model must arise from strings, and that therefore such a marvellous mechanism must exist. The only problem is that...it has not been discovered yet.

So, for the moment, the best we can do is...keep trying!, i.e. to use the experimental results available (such as $S U(3) \times S U(2)_{L} \times U(1)_{Y}$, three families, fermion masses, mixing angles, etc.), to discard models. Although the model space is in principle huge, a detailed analysis can reduced this to a reasonable size. For example, within the $Z_{3}$ orbifold with two Wilson lines, one can construct in principle a number of order 50000 of three-generation models with the $S U(3) \times S U(2) \times U(1)^{5}$ gauge group associated to the first $E_{8}$ of the heterotic string. However, a study implied that most of them are equivalent 29, and in fact, at the end of the day, only 192 different models were found 3029 . This reduction is remarkable, but we should keep in mind that the analysis of each one of these models is painful.

In summary, to obtain a connection between (string) theory and present (standard model) experiments is possible in principle but difficult in practice. But, what about future experiments (such as LHC)? Well, if Nature is supersymmetric at the weak scale, as many particle physicists believe (ironically string phenomenologists, at least some of them, who were originally supersymmetry phenomenologists, are not so enthusiastic nowadays with supersymmetry as in the past because of the recent developments), eventually the spectrum of supersymmetric particles will be measured providing us with a possible connection with the high-energy world of superstrings. Let us recall that in superstring constructions there is a natural hidden sector built in, the singlet fields $S$ and $T_{i}$ mentioned above, and that the Kähler potential $K\left(S, S^{*}, T_{i}, T_{i}^{*}\right)$ and the gauge kinetic function $f\left(S, T_{i}\right)$ of the fourdimensional supergravity Lagrangian are known. As a consequence, the soft supersymmetry-breaking terms, scalar masses $m_{\alpha}$, gaugino masses $M_{a}$, etc., can be computed in principle and compared with the experimentally observed supersymmetric spectrum 31 , i.e. we will be able to do what one could call 32 "Soft Phenomenology".

Although this approach will not probably be sufficient to select the superstring standard model, at least it will allow us to discard many constructions not producing the correct values for the soft terms. In addition, if experimen-

paper: submitted to World Scientific on January 3, 2019 
Figure 4. Curious scientist opening the Padora's box of D-brane constructions. Multitude of 'plagues' for hapless string phenomenologists escape.

talists find some extra particles which arise naturally in a particular string framework 33 , this might be helpful.

In any case we should not forget that the cosmological constant contributes to the value of the soft terms, introducing in principle another problem in our discussion. First of all, we have a new free parameter in the computation, e.g. $m_{\alpha}^{2} \sim m_{3 / 2}^{2}+V_{0} / M_{P}^{2}$. Second, as is well known, in any theory including gravity the natural value of the cosmological constant is huge (in our case $V_{0} \sim m_{3 / 2}^{2} M_{P}^{2}$ once supersymmetry is broken), and this is one of the biggest problems in particle physics. If we use a specific mechanism for the breaking of supersymmetry, all this may be specially disturbing, e.g. in gaugino condensation $V_{0}$ turns out to be negative and including this contribution one might obtain $m_{\alpha}^{2}<0$.

Until recently, the $E_{8} \times E_{8}$ heterotic superstring framework discussed above was thought as the only way in order to construct realistic string models. However, in the late nineties it has been discovered that D-brane configurations from string vacua or heterotic M-theory can also give rise to explicit models, with interesting phenomenological properties 3435 (although with unrealistic Yukawas for the moment, as in the case of the perturbative heterotic models). Of course, this means that we have more work to do since we have more models to analyze, but also... that the Pandora's box opened. As you know, Jupiter had crammed into a box all the diseases, sorrows, vices that afflict poor humanity. Pandora, the first woman, who did not know this, was seized with an eager curiosity to know what the box contained. One day she slipped off the cover and...forthwith there escaped all plagues for hapless man In our

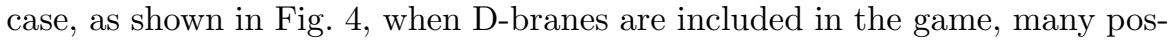
sibilities completely forbidden in the context of the heterotic string are now allowed: Non-supersymmetric models can be constructed $[36$, the string scale $M_{\text {string }}$ may be anywhere between the weak scale $M_{W}$ and the Planck scale $M_{\text {Planck }}$ 37, large extra dimensions are possible 38 , etc. Exaggerating, now the question is not the old one: What is possible to predict in string theory?, but, What is not possible to predict?

Let me point out, however, that some of these possibilities imply a hierarchy problem. For example, embedding the standard model inside D3-branes, 
Table 3. Statistics of the meeting String Phenomenology 2003, concerning the subjects of the talks.

\begin{tabular}{|c|c|}
\hline Subjects & Number of talks \\
\hline Branes & 17 \\
\hline Heterotic string & 8 \\
\hline M-theory & 7 \\
\hline Supersymmetry & 7 \\
\hline Cosmology & 5 \\
\hline $\mathrm{D}=5$ constructions & 3 \\
\hline Accelerators & 2 \\
\hline Quantum gravity & 1 \\
\hline AdS & 1 \\
\hline Non-commutative & 1 \\
\hline Electroweak & 1 \\
\hline Neutrinos & 1 \\
\hline$\ldots$ & $\ldots$ \\
\hline
\end{tabular}

one has

$$
\frac{M_{\text {string }}^{4}}{M_{c}^{3}}=\frac{\alpha M_{\text {Planck }}}{\sqrt{2}}=3.5 \times 10^{17} \mathrm{GeV},
$$

where $\alpha$ is the gauge coupling and $M_{c}$ is the compactification scale in the case of an overall modulus $T$. Thus one gets $M_{\text {string }} \approx 10^{11} \mathrm{GeV}$ much smaller than the Planck scale with $M_{c} \approx 10^{9} \mathrm{GeV}$, i.e. an apparently modest input hierarchy. However, in fact those values would imply

$$
S=\frac{1}{\alpha} \simeq 24, \quad T=\frac{1}{\alpha}\left(\frac{M_{\text {string }}}{M_{c}}\right)^{4} \simeq 10^{9}
$$

Thus one has a hierarchy problem but with the VEVs of the fields that one has to determine dynamically. Of course, if we want to lower $M_{\text {string }}$ further the hierarchy problem is worse. E.g. using eq. (10) for the case of two different compact dimensions $M_{1} \sim 10^{-13} \mathrm{GeV}$ (i.e. 1 millimeter) and $M_{2}=M_{3} \sim 10^{4}$ $\mathrm{GeV}$, one obtains $M_{\text {string }}=\left(M_{1} M_{2}^{2} \times 3.5 \times 10^{17} \mathrm{GeV}\right)^{1 / 4} \sim 1 \mathrm{TeV}$. But then,

$$
S=\frac{1}{\alpha} \simeq 24, T_{2}=\frac{1}{\alpha}\left(\frac{M_{\text {string }}^{4}}{M_{1}^{2} M_{3}^{2}}\right) \simeq 10^{31}, T_{1}=\frac{1}{\alpha}\left(\frac{M_{\text {string }}^{4}}{M_{2}^{2} M_{3}^{2}}\right) \simeq 10^{-3}
$$

In any case, it is clear that nowadays these constructions are the new super-stars, and that supersymmetry, Planck scale physics, small extra dimensions, or anything not involving D-branes is...out of fashion! Please, do not feel attack if you still work in these out-of-fashion issues... Of course I am 
Figure 5. Evolution: from the monkey to the string phenomenologist.

Figure 6. A string phenomenologist searching the standard model in 1985.

Figure 7. The huge number of possibilities in the model space that a string phenomenologist found in the analysis in 1985.

joking and exaggerating, and in fact after following the talks of this meeting my impression is that (although clearly the winners are the Branes) the 'old' constructions, such as the perturbative heterotic string, are having a revival. See Table 3 for more details.

Finally, given the previous discussion, let me summarize the evolution of string phenomenology using a few figures. First of all, the evolution from the monkey to the string phenomenologist is shown in Fig. 5 As is well known, the string phenomenologist is a fellow who spends most of the time using her/his computer compulsively: Sending revised versions to hep-ph (and even to hepth), complaining about references, preparing talks... Fortunately, from time to time she/he has a small hole in her/his tight schedule and spend some time thinking about string phenomenology. This is precisely the moment shown in Fig. 6] Clearly, the poor guy sit down at the front of the room is a string phenomenologist. In 1985 he really believed that the standard model was somewhere around him and that he would be able to find it. The problem, as you can see, is that the search of the standard model in strings is like to look a needle in a haystack. What about the guy at the back of the office? Well, he is clearly a string theoretician. As you can see he seems quite confident having a look to his very nice classification of string theories, $E_{8} \times E_{8}$ heterotic, type I, type IIA, ..., and with all the machinery to work with them in the shelves. But what for this theoretician is a nice classification, for the phenomenologist is a horrible nightmare. He has a incredible mess, a jungle, in his hands. As shown in Fig. 7 because of the huge number of models, each one with its own characteristics, the situation was, in a sense, depressing.

Now, we can see the evolution in the search of the standard model comparing Figs. 6 and 8 In the latter we see the same string phenomenologist but in 2003. Do you see any difference? Clearly, the mess is exactly the same: 
Figure 8. Evolution of string phenomenology: The same string phenomenologist searching the standard model as in Fig. [6] but in 2003. Do you see any evolution?

Figure 9. Because of the recent developments, the number of possibilities in 2003 is now bigger than in 1985 (see Fig [7): the nightmare is even worse!

terrible $^{c}$. Wait a moment, this is not true, as shown in Fig. 9] the mess is even worse! Because of the recent developments, the number of possible models is now bigger, and therefore the number of different results increases.

Anyway, the meeting is finishing, you are going back home, and I do not want you to leave Durham crying and depressed. So, let me tell you something very optimistic about string phenomenology. Not only the topic: from another viewpoint this situation is good because this means that still there is a lot work to do for newcomers. I am in the position of telling you something much stronger. Usually people says that the problem of strings is that they do not have a clear prediction that can be tested. On the contrary, I can tell you very proudly that predictions can be done. Indeed, I did an important prediction using strings two years ago. This prediction has been finally fulfilled. The British experimentalists have tested it very recently. Please, have a look to Fig. 10] where the abstract and date of the article containing the prediction is shown. Thank you very much for your attention.

\section{Acknowledgments}

I thank the organizers of this wonderful conference in Durham during July 29-August 4, 2003, for suggesting me the idea of giving an 'entertaining' talk about string phenomenology. At least I tried.

The photograph shown in Figs. 6 and 8 is a work of art made by Jeff Wall in 1994, with the title "Untangling". Although several colleagues thought that I was the author, I only deserve the reputation of the modifications shown in Figs. 7 and 9. When I first saw this photograph in October 1999, in an exhibition of the Pamela and Richard Kramlich collection of media art at San Francisco Museum of Modern Art, I immediately thought 'This is the best

${ }^{c}$ An optimistic view of this situation was pointed out to me by the experimentalist H.U. Martyn. In his opinion, working in string phenomenology is healthy because 18 years have gone by between the two photographs and the guy does not look old at all!

paper: submitted to World Scientific on January 3, 2019 


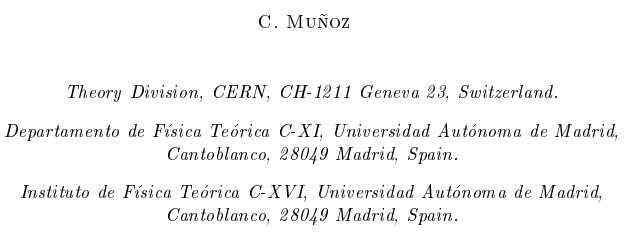

\section{Abstract}

Assuming that the standard model of particle physics arises from type IIB compactified on an orientifold with D5-branes intersecting at angles on $T^{4}$, we predict that David Beckham will play in Real Madrid in 2003. As a by-product our analysis implies that David and Victoria Beckham live at the intersections.

Figure 10. First page of an article containing a clear prediction from strings, that has been fulfilled.

possible summary of the state-of-the-art in string phenomenology'.

\section{References}

1. F.J. Dyson, 'Field Theory', article reproduced in page 17 of the book Particles and Fields (Scientific American, 1980).

2. For recent reviews, with a complete list of the historical works contributing to the formulation of the standard model, see J.L. Rosner, 'The standard model in 2001', hep-ph/0108195 'Resource letter: the standard model and beyond', Am. J. Phys. 71 (2003) 302 hep-ph/0206176.

3. J. Scherk and J.H. Schwarz, 'Dual models for nonhadrons', Nucl. Phys. B81 (1974) 118;

T. Yoneya, 'Connection of dual models to electrodynamics and gravidynamics', Prog. Theor. Phys. 51 (1974) 1907. 
4. M.B. Green and J.H. Schwarz, 'Anomaly cancellation in supersymmetric $\mathrm{D}=10$ gauge theory and superstring theory', Phys. Lett. B149 (1984) 117.

5. D. Gross, J. Harvey, E. Martinec and R. Rohm, 'The heterotic string', Phys. Rev. Lett 54 (1985) 502; 'Heterotic string theory. 1. The free heterotic string', Nucl. Phys. B256 (1985) 253; 'Heterotic string theory. 2. The interacting heterotic string', Nucl. Phys. B267 (1986) 75.

6. P. Candelas, G.T. Horowitz, A. Strominger and E. Witten, 'Vacuum configurations for superstrings', Nucl. Phys. B258 (1985) 46.

7. L.J. Dixon, J. Harvey, C. Vafa and E. Witten, 'Strings on orbifolds', Nucl. Phys. B261 (1985) 678; 'Strings on orbifolds 2', Nucl. Phys. B274 (1986) 285.

8. H. Kawai, D.C. Lewellen and S.H.H. Tye, 'Construction of fourdimensional fermionic string models', Phys. Rev. Lett. 57 (1986) 1832, Erratum, ibid. 58 (1987) 429; 'Construction of fermionic string models in four dimensions' Nucl. Phys. B288 (1987) 1;

I. Antoniadis, C. Bachas and C. Kounnas, 'Four-dimensional superstrings', Nucl. Phys. B289 (1987) 87.

9. B. Greene, K.H. Kirklin, P.J. Miron and G.G. Ross, 'A superstring inspired standard model', Phys. Lett. B180 (1986) 69; 'A three generation superstring model. 1. Compactification and discrete symmetries', Nucl. Phys. B278 (1986) 667; 'A three generation superstring model. 2. Symmetry breaking and the low-energy theory', Nucl. Phys. B292 (1987) 602.

10. J.A. Casas and C. Muñoz, 'Three generation $S U(3) \times S U(2) \times U(1)_{Y} \times$ $U(1)$ orbifold models through Fayet-Iliopoulos terms', Phys. Lett. B209 (1988) 214; 'Three generation $S U(3) \times S U(2) \times U(1)_{Y}$ models from orbifolds', Phys. Lett. B214 (1988) 63; 'Yukawa couplings in $S U(3) \times$ $S U(2) \times U(1)_{Y}$ orbifolds models', Phys. Lett. B212 (1988) 343;

A. Font, L.E. Ibáñez, H.P. Nilles and F. Quevedo, 'Yukawa couplings in degenerate orbifolds: towards a realistic $S U(3) \times S U(2) \times U(1)$ superstring', Phys. Lett. B210 (1988) 101.

11. I. Antoniadis, J. Ellis, J.S. Hagelin and D.V. Nanopoulos, 'GUT model building with fermionic four-dimensional strings', Phys. Lett. B205 (1988) 459; 'An improved $S U(5) \times U(1)$ model from four-dimensional string', Phys. Lett. B208 (1988) 209, Addendum, ibid. B213 (1988) 562 ; 'The flipped $S U(5) \times U(1)$ string model revamped', Phys. Lett. B231 (1989) 65;

A. Faraggi, D.V. Nanopoulos and K. Yuan, 'A standard like model in the 4-D free fermionic string formulation', Nucl. Phys. B335 (1990) 347.

paper: submitted to World Scientific on January 3, 2019 
12. L.E. Ibáñez, H.P. Nilles and F. Quevedo, 'Orbifolds and Wilson lines', Phys. Lett. B187 (1987) 25.

13. L.E. Ibáñez, J.E. Kim, H.P. Nilles and F. Quevedo, 'Orbifolds compactifications with three families of $S U(3) \times S U(2) \times U(1)^{n}$, Phys. Lett. B191 (1987) 3.

14. L.E. Ibáñez, J. Mas, H.P. Nilles and F. Quevedo, 'Heterotic strings in symmetric and asymmetric orbifold backgrounds' Nucl. Phys. B301 (1988) 157.

15. J.A. Casas, E.K. Katehou and C. Muñoz, ' $U(1)$ charges in orbifolds: anomaly cancellation and phenomenological consequences', Nucl. Phys. B317 (1989) 171.

16. E. Witten, 'Some properties of $O(32)$ superstrings', Phys. Lett. B149 (1984) 351;

M. Dine, N. Seiberg and E. Witten, 'Fayet-Iliopoulos terms in string theory', Nucl. Phys. B289 (1987) 317;

J.J. Atick, L.J. Dixon and A. Sen, 'String calculation of Fayet-Iliopoulos D terms in arbitrary supersymmetric compactifications', Nucl. Phys. B292 (1987) 109;

M. Dine, I. Ichinose and N. Seiberg, 'F terms and D terms in string theory', Nucl. Phys. B293 (1987) 253.

17. S. Hamidi and C. Vafa, 'Interactions on orbifolds', Nucl. Phys. B279 (1987) 465 ;

L.J. Dixon, D. Friedan, E. Martinec and S. Shenker, 'The conformal field theory of orbifolds', Nucl. Phys. B282 (1987) 13.

18. L.E. Ibáñez, 'Hierarchy of quark-lepton masses in orbifolds superstring compactification', Phys. Lett. B181 (1986) 269.

19. J.A. Casas and C. Muñoz, 'Fermion masses and mixing angles: a test for string vacua', Nucl. Phys. B332 (1990) 189, Erratum, ibid. B340 (1990) 280 ;

J.A. Casas, F. Gómez and C. Muñoz, 'Fitting the quark and lepton masses in string theories', Phys. Lett. B292 (1992) 42 hep-th/9206083.

20. S.A. Abel and C. Muñoz, 'Quark and lepton masses and mixing angles from superstring constructions', J. High Energy Phys. 02 (2003) 010 hep-ph/0212258.

21. N. Cabibbo, 'Unitary symmetry and leptonic decays', Phys. Rev. Lett. 10 (1963) 531;

M. Kobayashi and T. Maskawa, ' $\mathrm{CP}$ violation in the renormalizable theory of weak interaction', Prog. Theor. Phys. 49 (1973) 652.

22. PARTICLE DATA GROUP collaboration, K. Hagiwara et al., 'Review of particle physics', Phys. Rev. D66 (2002) 010001. 
23. T. Kobayashi and O. Lebedev, 'Heterotic string backgrounds and CP violation', Phys. Lett. B565 (2003) 193 hep-th/0304212.

24. J.E. Kim and H.B. Kim, 'An orbifold compactification with three families from twisted sectors', Phys. Lett. B300 (1993) 343 hep-ph/9212311;

G. Aldazabal, A. Font, L.E. Ibáñez and A.M. Uranga, 'Building GUTs from strings', Nucl. Phys. B465 (1996) 34 hep-th/9508033;

J. Giedt, 'Spectra in standard-like $Z_{3}$ orbifold models', Ann. Phys. 297 (2002) 67 hep-th/0108244;

J.E. Kim, ' $Z_{3}$ orbifold construction of $S U(3)^{3}$ GUT with $\sin ^{2} \theta_{W}^{0}=3 / 8$ ', Phys. Lett. B564 (2003) 35 hep-th/0301177; 'Three family $Z_{3}$ orbifold trinification, MSSM and doublet-triplet splitting problem', Phys. Lett. B567 (2003) 87 hep-ph/0305002; ' $S U(3)$ trits of orbifolded $E_{8} \times E_{8}^{\prime}$ heterotic string and supersymmetric standard model', J. High Energy Phys. 08 (2003) 010 hep-ph/0308064.

25. S. Chaudhuri, S.-W. Chung, G. Hockney and J. Lykken, String consistency for unified model building', Nucl. Phys. B456 (1995) 89 hep-ph/9501361;

S. Chaudhuri, G. Hockney and J. Lykken, 'Three generations in the fermionic construction', Nucl. Phys. B469 (1996) 357 hep-th/9510241; G.B. Cleaver, A. Faraggi and D.V. Nanopoulos, 'String derived MSSM and M-theory unification', Phys. Lett. B455 (1999) 135 hep-ph/9811427;

G.B. Cleaver, A. Faraggi, D.V. Nanopoulos and J.W. Walker, 'Phenomenology of nonabelian flat directions in a minimal superstring standard model', Nucl. Phys. B620 (2002) 259 hep-ph/0104091.

26. Z. Maki, M. Nakagawa and S. Sakata, 'Remarks on the unified model of elementary particles', Prog. Theor. Phys. 28 (1962) 870.

27. For recent reviews, see for example J.W.F. Valle, 'Neutrinos: summarizing the state-of-the-art', hep-ph/0205216

M.C. Gonzalez-Garcia, 'Neutrino masses and mixing: where we stand and where we are going', hep-ph/0211054

28. C. Muñoz, 'Supersymmetry breaking in string theories', talk given at the Workshop on Phenomenology in High Energy Physics and Cosmology, ICTP, Trieste, July 26-27, 1990.

29. J.A. Casas, M. Mondragon and C. Muñoz, 'Reducing the number of candidates to standard model in the $Z_{3}$ orbifold', Phys. Lett. B230 (1989) 63.

30. J. Giedt, 'Completion of standard-like embeddings', Ann. Phys. (NY) B289 (2001) 251 hep-th/0009104.

31. For a review, see A. Brignole, L.E. Ibáñez and C. Muñoz, 'Soft 
supersymmetry-breaking terms from supergravity and superstring models', in the book 'Perspectives on Supersymmetry', Ed. G. Kane, World Scientific (1998) 125 hep-ph/9707209.

32. C. Muñoz, 'Soft Phenomenology', Proceedings of the International Europhysics Conference (EPS) on High-Energy Physics, 1997 (SpringerVerlag, Berlin, 1999) hep-ph/9710388.

33. See e.g. C. Muñoz, 'A kind of prediction from superstring model building', J. High Energy Phys. 12 (2001) 015 hep-th/0110381, and references therein.

34. C. Angelantonj, M. Bianchi, G. Pradisi, A. Sagnotti and Y. Stanev, 'Chiral asymmetry in four-dimensional open-string vacua', Phys. Lett. B385 (1996) 96 hep-th/9606169;

M. Berkooz and R.G. Leigh, 'A $\mathrm{D}=4 \mathrm{~N}=1$ orbifold of type I strings', Nucl. Phys. B483 (1997) 187 hep-th/9605049;

M. Berkooz, M.R. Douglas and R.G. Leigh, 'Branes intersecting at angles', Nucl. Phys. B480 (1996) 265 hep-th/9606139;

Z. Kakushadze, 'A three-family SU(6) Type I compactification', Phys. Lett. B434 (1998) 269 hep-th/9804110;

J. Lykken, E. Poppitz and S.P. Trivedi, 'Branes with GUTs and supersymmetry breaking', Nucl. Phys. B543 (1999) 105 hep-th/9806080;

Z. Kakushadze and S.-H.H. Tye, 'Three generations in type I compactifications' Phys. Rev. D58 (1998) 126001 hep-th/9806143;

M. Cvetic, M. Plumacher and J. Wang, 'Three family type IIB orientifold string vacua with non-Abelian Wilson lines', J. High Energy Phys. 04 (2000) 004 hep-th/9911021;

G. Aldazabal, L.E. Ibáñez, F. Quevedo and A.M. Uranga, 'D-Branes at singularities : A bottom-up approach to the string embedding of the standard model', J. High Energy Phys. 08 (2000) 002 hep-th/0005067;

M. Cvetic, G. Shiu and A.M. Uranga, 'Three-family supersymmetric standard-like models from intersecting brane worlds', Phys. Rev. Lett. 87 (2001) 201801 hep-th/0107143;

D. Bailin, G.V. Kraniotis and A. Love, 'Supersymmetric Standard Models on D-Branes', Phys. Lett. B502 (2001) 209 hep-th/0011289.

35. R. Donagi, A. Lukas, B.A. Ovrut and D. Waldram, 'Holomorphic vector bundles and non-perturbative vacua in M-Theory', J. High Energy Phys. 06 (1999) 034 hep-th/9901009;

R. Donagi, B.A. Ovrut and D. Waldram, 'Moduli spaces of fivebranes on elliptic Calabi-Yau threefolds', J. High Energy Phys. 11 (1999) 030 hep-th/9904054;

R. Donagi, B.A. Ovrut, T. Pantev and D. Waldram, 'Standard mod-

paper: submitted to World Scientific on January 3, 2019 
els from heterotic M-theory', Adv. Theor. Math. Phys. 5 (2002) 93 hep-th/9912208;

A.E. Faraggi, R. Garavuso and J.M. Isidro, 'Nonperturbative flipped SU(5) vacua in heterotic M-theory', Nucl. Phys. B641 (2002) 111 hep-th/0204080;

B.A. Ovrut, T. Pantev and R. Reinbacher, 'Invariant homology on standard model manifolds', hep-th/0303020

36. R. Blumenhagen, L. Görlich, B. Körs and D. Lüst, 'Noncommutative compactifications of type I strings on tori with magnetic flux', J. High Energy Phys. 10 (2000) 006 hep-th/0007024; 'Magnetic flux in toroidal type I compactification', Fortsch. Phys. 49 (2001) 591 hep-th/0010198; G. Aldazábal, S. Franco, L.E. Ibáñez, R. Rabadán and A.M. Uranga,'D $=4$ chiral string compactifications from intersecting branes', J. Math. Phys. 42 (2001) 3103 hep-th/0011073;

R. Blumenhagen, B. Körs and D. Lüst, 'Type I strings with F- and Bflux', J. High Energy Phys. 02 (2001) 030 hep-th/0012156;

L.E. Ibáñez, F. Marchesano and R. Rabadan, 'Getting just the standard model at intersecting branes', J. High Energy Phys. 11 (2001) 002 hep-th/0105155;

R. Blumenhagen, B. Kors, D. Lust and T. Ott, 'The standard model from stable intersecting brane world orbifolds', Nucl. Phys. B616 (2001) 3 hep-th/0107138;

C. Kokorelis, 'New standard model vacua from intersecting branes', $J$. High Energy Phys. 09 (2002) 029 hep-th/0205147.

37. J.D. Lykken, 'Weak Scale Superstrings', Phys. Rev. D54 (1996) 3693 hep-th/9603133;

E. Witten, 'Strong Coupling Expansion Of Calabi-Yau Compactification', Nucl. Phys. B471 (1996) 135 hep-th/9602070;

38. N. Arkani-Hamed, S. Dimopoulos and G.R. Dvali, 'The hierarchy problem and new dimensions at a millimeter', Phys. Lett. B429 (1998) 263 hep-ph/9803315;

I. Antoniadis, N. Arkani-Hamed, S. Dimopoulos and G.R. Dvali, 'New dimensions at a millimeter to a Fermi and superstrings at a TeV', Phys. Lett. B436 (1998) 257 hep-ph/9804398;

I. Antoniadis, 'A possible new dimension at a few TeV', Phys. Lett. 246B (1990) 377. 
This figure "azores2.jpg" is available in "jpg" format from: http://arxiv.org/ps/hep-ph/0312091v2 
This figure "cuerdas.jpg" is available in "jpg" format from: http://arxiv.org/ps/hep-ph/0312091v2 
This figure "cuerdasconflechas.jpg" is available in "jpg" format from: http://arxiv.org/ps/hep-ph/0312091v2 
This figure "cuerdasconflechas2.jpg" is available in "jpg" format from: http://arxiv.org/ps/hep-ph/0312091v2 
This figure "evolution.jpg" is available in "jpg" format from: http://arxiv.org/ps/hep-ph/0312091v2 
This figure "pandoraletras.jpg" is available in "jpg" format from: http://arxiv.org/ps/hep-ph/0312091v2 
This figure "puzzle.jpg" is available in "jpg" format from: http://arxiv.org/ps/hep-ph/0312091v2 
This figure "puzzle2.jpg" is available in "jpg" format from: http://arxiv.org/ps/hep-ph/0312091v2 